\title{
Adipose Tissue Graft Improves Early but not Late Stages of Nerve Regeneration
}

\author{
VLAD BLOANCĂ ${ }^{1}$, AMALIA RALUCA CEAUȘU ${ }^{2}$, ANDREEA ADRIANA JITARIU ${ }^{2}$, \\ ARIANA BARMAYOUN ${ }^{3}$, RALUCA MOS ${ }^{3}$, ZORIN CRĂINICEANU $^{1}$ and TIBERIU BRATU ${ }^{1}$ \\ Departments of ${ }^{1}$ Plastic Surgery and ${ }^{2}$ Microscopic Morphology/Histology, and \\ ${ }^{3}$ Angiogenesis Research Center, Victor Babes University of Medicine and Pharmacy, Timișoara, Romania
}

\begin{abstract}
Aim: The aim of the study was to assess the effect of autologous fat graft on nerve regeneration by means of immunohistochemistry. Materials and Methods: The rat sciatic nerve was used; complete transection followed by primary neurorrhaphy was performed on both hind legs, on the left side a processed fat graft was applied, surrounding the nerve. Nerve biopsies were collected and immunohistochemical procedures were performed for glial fibrillary acidic protein (GFAP) and for neurofilamentassociated protein(NFAP). Results: At 4 weeks, GFAPpositive cells were observed in the connective tissue formed between the two nerve endings on the left side only. At 10 weeks, GFAP-positive structures were present and exhibited a tendency to become linear on both sides, with an increased density on the left. NFAP-positive expression was present in the left treated limb with a disorganized pattern. Conclusion: Adipose tissue led to the stimulation of GFAP-positive Schwann cells, which could have a positive impact on nerve regeneration in the clinical setting.
\end{abstract}

Peripheral nerve injuries are a challenge for both the surgeon and the clinician, a challenge supported by the absence of evaluation markers for post-traumatic healing. This challenge is maintained due to the structural particularities and the difficulties of peripheral nerve biopsy sampling following surgical intervention. Currently, no serum marker that could be used as an evaluation biomarker for nerve regeneration is available; moreover; the correlation between motor rehabilitation and the morphological sequences that form the

This article is freely accessible online.

Correspondence to: Zorin Crăiniceanu, Department of Plastic Surgery, Victor Babes University of Medicine and Pharmacy, Timișoara, Romania, E. Murgu 2, 300041, Timișoara, Timiș, Romania. E-mail: zcrainiceanu@gmail.com

Key Words: Nerve, fat tissue addition, GFAP, NFAP. basis of this recovery have been very little studied and are poorly understood, most of them being rarely reported in experimental models of nerve regeneration.

Protein S100 is specific and able to identify mature Schwann cells; neurofilament associated protein (NFAP) is applied for the identification of intra-axonal intermediate filaments. In the study of nerve regeneration, glial fibrillary acidic protein (GFAP) seems to mark reactive Schwann cells and is normally expressed only in the glial cells of the central nervous system. Very few clinical data and case reports are available regarding nerve regeneration tested through usage of GFAP. In pathology, GFAP is expressed in peripheral nerve sheath tumors (malignant schwannomas) (1). Recent studies that used rat experimental models have shown that differentiated Schwann-like stem cells from adipose tissue (AdSC) were positive for GFAP expression. Study of their morphological features have demonstrated that these AdSC-derived GFAP-positive cells exhibited a bipolar, tripolar and even multipolar structure, presented a single layer growth pattern, but were characterized by a flattened morphology, large dimensions and thin expansions (2). The majority of these cells proved to be highly proliferative in the aforementioned study, the percentage of such differentiated cells from the adipose tissue was over 95\% (3). This study did not establish a correlation between the percentage of Schwann-like GFAP-positive cells and muscle volume restoration. Regarding this aspect, we considered the utility of the evaluation of the presence as well as the distribution of corresponding GFAP-positive cells in the muscle, taking into account that such an evaluation has not yet been reported in the scientific literature.

NFAP marks intermediate filaments located in the inner part of a severed axon, thus being useful in the evaluation of postinjury nerve regeneration. This marker is not specific for regenerated filaments, but does represent a useful tool in the evaluation of nervous dynamic regeneration. The use of NFAP was reported in several studies, and was regarded as a quantitative marker for the rapidity of nerve regeneration in an inflammatory context (4), but also as an immunohistochemical 
marker for nerve regeneration in different experimental models concerning nerve gaps and the use of nerve conduits (5-7). We did not find any literature studies referring to the quantification of NFAP-positive filaments in regenerated nerves and in corresponding muscle(s).

\section{Materials and Methods}

Rat model. An experimental rat model was designed based on a group of 10 Wistar rats. We organized the animals into two groups: a control group of two animals and a treated group with eight animals (designated S1-S8). In the treated group, a complete transection of the sural nerve was performed on the left and right hind limbs of each animal followed by direct neurorrhaphy alone of the right hind limb and by direct neurorrhaphy and addition of processed fat tissue around it for the left hind limb. Briefly, fat tissue was harvested from inguinal area of the same rat, centrifuged $3 \mathrm{~min}$ at $1,008 \times \mathrm{g}$, with separation of three layers, from top to bottom: oil, processed fat and the fluid portion. We used the processed fat to cover the left neurorrhaphy. After 4 weeks, four animals were sacrificed and nerve and adjoining muscle biopsies were collected. The other four animals were sacrificed at 10 weeks from the beginning of the study, followed by the collection of the right and left treated sural nerves, with the respective gastrocnemius muscle.

Tissue processing. The tissue specimens were fixed in $10 \%$ buffered formalin for $24 \mathrm{~h}$, followed by routine paraffin embedding. Threemicrometer serial sections were prepared and one slide for each case was stained with hematoxylin and eosin for histopathological diagnosis. Corresponding slides for each case were immunostained for GFAP and NFAP (Leica Microsystems, Wetzlar, Germany). All immunohistochemical procedures were performed in a fully automated manner by Bond Max System (Leica Microsystems, Wetzlar, Germany).

Microscopic analysis, data and image acquisitions. Conventional microscopy was performed for the interpretation of morphology and immunohistochemical stained slides by an Axio Zoom Image A2 Research Microscope (Zeiss, Jena, Germany). Images were captured using an AxioCam 506 Color attached to the microscope and transferred to the computer for image analysis with ZEN program kindly provided by Zeiss (Zeiss, Jena, Germany).

\section{Results}

Control group. The animals of the control group exhibited GFAP-positive structures characterized by a relatively organized distribution, corresponding to the periaxonal zones, related to the Schwann cells, but also NFAP-positive structures with a continuous aspect, corresponding to the neurite. In addition, at the muscular level, GFAP staining showed isolated expression that was restricted to the areas between the muscle fibers, presenting a discontinuous, dotted aspect, strongly associated with the muscle fibers and a low density of GFAP-positive structures (Figure 1a and b). NFAP staining identified nerve threads present in an organized layout amongst the muscle fibers (Figure 1c and d).
Treated group at 4 weeks. For the right limb, the neurorrhaphy zone presented a disruption of the GFAPpositive cells and heterogeneous staining, GFAP-positive structures were organized as notch-like structures discontinuously located amongst the affected nerve fibers. NFAP staining established the difference between the used specimens as following: in the right limb of rat $\mathrm{S} 1$, we noted the absence of the linear structures expressing NFAP, observed in the control group. For the right limb of rat S2, the proximal nerve ending contained rather elongated, linear structures, while in the distal ending, NFAP-positive structures exhibited a shorter length and marked discontinuities between the positive fragments. For rat S4, we identified the presence of NFAP-positive filaments between the nerve endings in the right limb. The muscle exhibited a similar density as that of the control group containing GFAP-positive cells, but NFAP staining highlighted the absence of filamentous structures at the level of the endoneurium, with the maintenance of its expression in the large nervous structures from the perineurium rat $\mathrm{S} 1$, and rat S2 at the level of the nervous thread adjacent to the muscle. NFAP expression was maintained and the linear structures were relatively continuous; in the inner part of the muscle, at the level of the endomysium, we did not identify any NFAP-positive structures. The nerve exhibited an increased density of GFAP-positive structures on the left side, either with a dotted pattern or dispersed into large groups. The density was higher in comparison to the control group and presented the the tendency to accumulate at the nerve endings. For the left limb of rat S1, GFAP-positive structures were also present in the connective tissue formed between the two nerve endings (Figure 2a). For rat S2, the GFAP positive structures were unequal in dimensions in the left limb, some being characterized by a linear pattern, others having variable length and an increased density, $57 /$ microscopic field (Figure $2 \mathrm{~b}$ and c). At the muscular level, we observed rare GFAP-positive structures, 1$2 /$ microscopic field in rat S1 and 20/microscopic field for rat $\mathrm{S} 2$, localized inside the muscle and in the endoneurium. These structures were persistent and presented a normal distribution in the cross-sectioned nerve at the level of the epimysium. The NFAP-positive linear structures were absent from the neurorrhaphy zone. We detected dotted-like, NFAPpositive structures with no obvious continuity between them, $19 /$ microscopic field for rat S1; for rat S2, we identified isolated NFAP-positive structures on longitudinal section and NFAP-positive aggregates, 11 linear structures/microscopic field. In the muscle, the density of NFAP-positive structures was increased and presented structures with larger dimensions in both the endomysium and in the epimysium, $5-7 /$ microscopic field for rat $S 1$. These structures were rarely identified in the left limb of rat $\mathrm{S} 2$. 


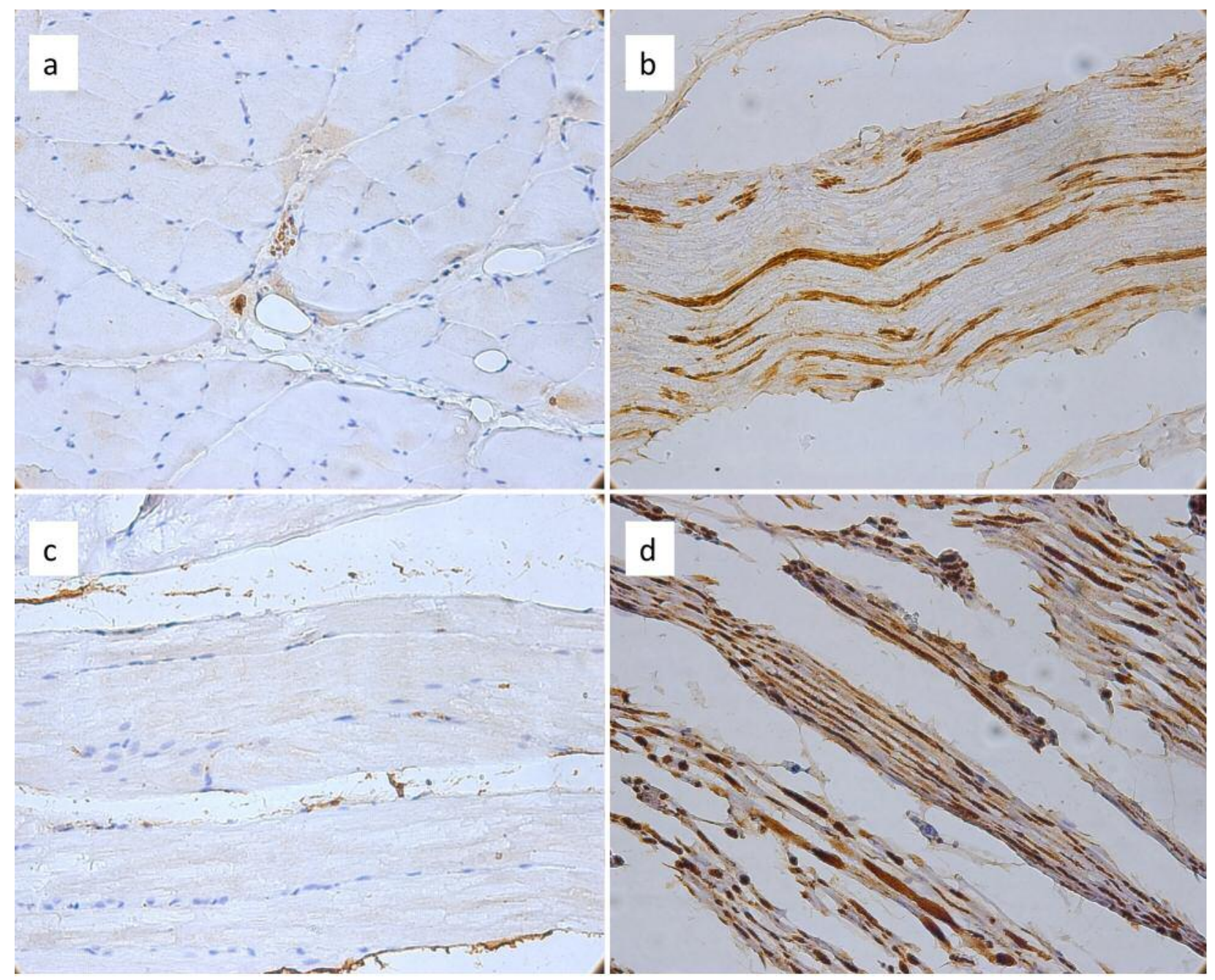

Figure 1. The control group exhibited normal glial fibrillary acidic protein (GFAP)-positive reaction at the muscle (a, magnification $\times 100)$ and at the nervous $(b$, magnification $\times 200)$ level. The features of neurofilament-associated protein (NFAP)-positive reaction are apparent at the muscular $(c$, magnification $\times 100)$, and at the nervous $(d$, magnification $\times 200)$ level.

Treated group at 10 weeks. At the nerve level, grouped GFAP-positive structures were present in the right limb of rat S5 and exhibited a tendency to become linear; the muscle presented a high density of GFAP-positive structures in the endomysium.

GFAP reaction was positive and isolated in the nerve of the right limb of rat S6, with a density that was similar to that of the previously examined slides. However, its expression was absent in the muscle. The positive reaction for GFAP detected in the nerve was characterized by the presence of discontinuous filaments, with increased density and by differences in the intensity of expression. Both the density and intensity of staining were weaker in the neurorrhaphy zone. Rare NFAP-positive threads were detected in the tissue that was interposed between the two nerve endings. At the muscular level, NFAP expression was positive but inconsistent and heterogeneously distributed. GFAP reaction was present in the left limb of rat S6 and was associated with an increased density but intensity of the reaction was reduced at the nerve level. The distribution observed at the muscular level was predominantly at the periphery of the muscle, occurring as large groups and amongst the muscle fibers. NFAPpositive threads were present in the peri-nervous adipose tissue and in the cross-sectioned nerve; the distribution was similar in the muscle containing GFAP-positive cells but we also noted the presence of NFAP-positive structures amongst the fascicles. 


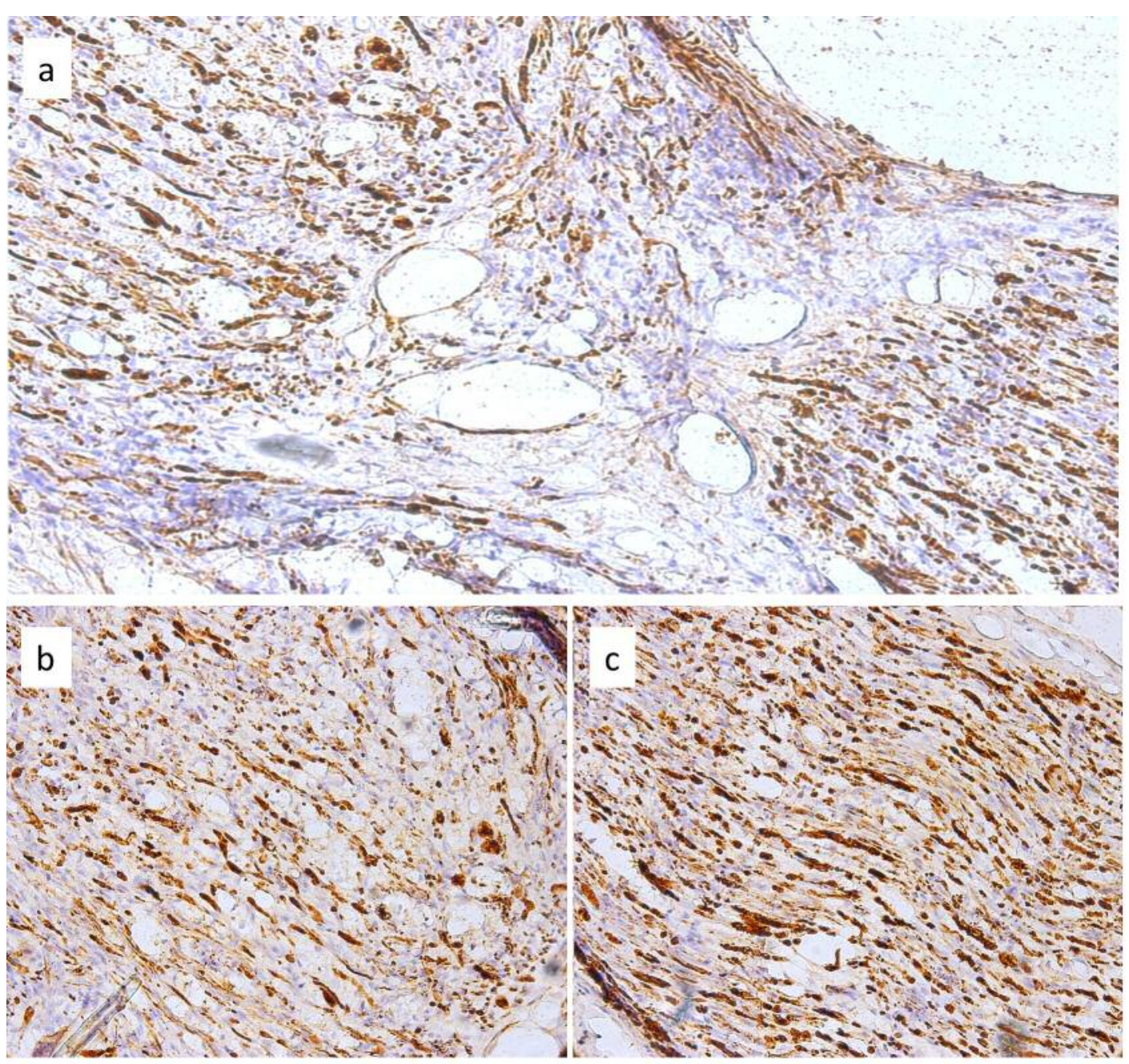

Figure 2. Adipose tissue-treated group. The continuity between the two segments of the sutured nerve is apparent. Note the detailed positive reaction for glial fibrillary acidic protein (GFAP) between the segments for the left limb of rat S1 (a, magnification $\times 400)$. Unequal dimensions and a heterogeneous distribution of GFAP-positive structures can be seen at the nerve level between the right (b, magnification $\times 200)$ and left $(c$, magnification $\times 200$ ) sural nerve of rat $S 2$.

The left limb of rat $\mathrm{S} 7$ presented regeneration in the neurorrhaphy zone, using reactions for both GFAP and NFAP. These reactions were characterized as aberrant and disorganized. We noted the presence of both horizontally and vertically disposed neurofilaments (Figure 3). In the right limb of rat S8, GFAP reaction was inconsistent and heterogeneous at the nervous level. The expression was predominantly peripheral at the muscular level. NFAP expression was low in the studied nerve and the neurite presented discontinuities. In the muscle, we observed 2-4 positive structures with a peripheral distribution along with numerous nervous threads.

In the left limb of rat $\mathrm{S} 8$, we found a positive reaction for GFAP in the neurorrhaphy zone which presented a similar density to that observed during the fourth week. However, the intensity of the reaction was low. In comparison to the proximal ending, the distal ending presented isolated nervous structures. The proximal ending of the nerve was characterized by the presence of a relatively organized and continuous layer of GFAP-positive structures; in addition, we noted that GFAP-positive structures located in the muscle had a peripheral distribution. NFAP reaction was isolated, dotted and presented an increased density in the tissue located between the two nervous endings.

\section{Discussion}

The treated tissue that was sampled at 4 weeks presented a disorganized, positive reaction for GFAP, at the nervous level, in the case of the sectioned nerve that was treated by means of neurorhaphy only (right side). At the level of the nerve treated 


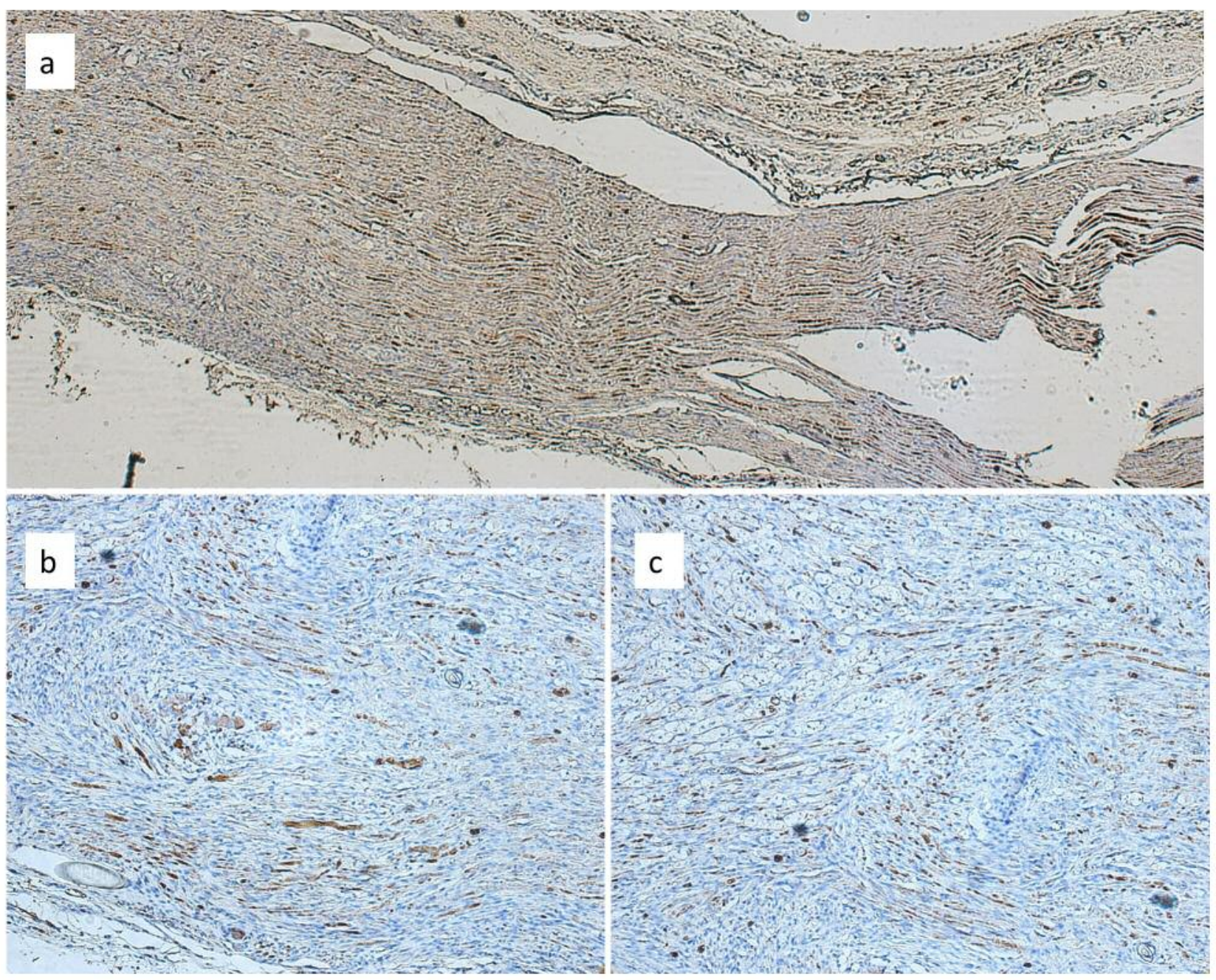

Figure 3. Adipose tissue-treated group. Regeneration is present but disorganized (a, magnification $\times 100$ ). The process was made evident through the use of staining for glial fibrillary acidic protein $(b$, magnification $\times 100)$ and neurofilament-associated protein $(c$, magnification $\times 100)$ of tissue from the left limb of rat $S 7$.

with addition of adipose tissue (left side), a positive reaction was also observed between the sectioned endings. Unlike in controls, the density of GFAP expression was increased, thus suggesting the activity of Schwann cells following trauma, with a pattern of disposition featuring groups or dots. NFAP reaction highlighted an incomplete regeneration process through the presence of discontinuous filaments. The treated group that was sampled at 10 weeks presented approximately the same density of GFAP reaction. However, the intensity was quite low, especially on the left side, where the structures exhibited a disrupted morphology and had the tendency to be organized and become linear. In other words, the degree of Schwann cell activation decreased (via modulation of the inflammatory response through the addition of processed adipose tissue). Regarding the regeneration process, the two endings presented zones of continuity but the neurofilaments were not completely organized, suggesting that the process was not yet defined at 10 weeks. We noted the existence of a previous remodeling stage.

These results represent the first microscopic aspects in evaluation of the differential expression of combined GFAP/ NFAP reactions in the early stages of nerve regeneration. The addition of adipose tissue led to the stimulation of GFAP-positive Schwann cells, as evidenced through the increase in the density and distribution of these cells.

Previous data regarding GFAP expression in reactive Schwann cells demonstrated that GFAP plays a major role in the re-innervation of the neuromuscular junction belonging to the muscle fibers innervated by the degenerated nerve after 13 days following injury (8). In the previously mentioned study, conducted by Berg et al., GFAP was 
analyzed along with vimentin, an aspect that does not have any specificity from our point of view and has a limited impact on the post-injury motor response. This fact was admitted by the authors themselves as a limitation of their study. However, GFAP remains a relatively specific marker for the quantification of reactive Schwann cells in the peripheral nervous system, its utility being increased through its association with NFAP applied in our study. We consider this association as a useful tool for the evaluation of both the degree of nervous rehabilitation and the functionality of the regenerated neurofilaments. Literature data are extremely limited and heterogeneous regarding the GFAP-NFAP tandem. Despite the fact that GFAP has been studied in peripheral nerves since 1992 (9), the role of GFAP-positive cells remains incompletely elucidated. Moreover, the quantification of GFAP-positive Schwann cells following the addition of fat tissue is even less well studied. In the previous mentioned study by Nona et al. (9), the fact that GFAP-positive Schwann cells are capable of appearing at both endings of the severed nerve was been demonstrated. This partially contradicts the current data regarding the histology of nerve regeneration.

Reactive GFAP-positive Schwann cells have been studied along their associated microenvironment in order to observe the possible influences on microenvironmental factors on Schwann cell reactivation and their ability to express GFAP. As examples of such research studies that refer to the microenvironmental influence, Knoops et al. used fibrin and fibronectin as support material for the regeneration of the peripheral nerve. This material seemed to stimulate peripheral nerve regeneration through stimulation of numerical growth of GFAP-positive cells (10). It seems that the induction of GFAP expression in reactive Schwann cells is mandatory for peripheral nerve regeneration. However, the molecular aspects and the factors that influence this process are incompletely understood. Amongst the factors that are implicated in this phenomenon, IL6 plays an important role in the signal transducer and activator of transcription 3 (STAT3)-mediated induction of GFAP expression (11). Recently, Li et al. published indirect data demonstrating an increase of serum IL6 level following human AdSC addition in a murine experimental model of autoimmune encephalomyelitis (12). The same study stated that transplantation of human AdSCs positively influenced the histological and functional changes of the affected nerves and reduced the inflammatory infiltrate and the level of demyelination. The results obtained by Li et al. partially overlap our data. In our study, the addition of fat tissue was followed by changes in the amount of perineural inflammatory infiltrate, starting with a decrease up to complete disappearance. These phenomena were accompanied by histological aspects of accelerated regeneration in the nerve that was treated with processed adipose tissue.
The IL6-STAT3 tandem that characterized the microenvironment induced by the adipose tissue addition mentioned above may also be affected by physical exercise, as mentioned by Ashour and collaborators (13). Ashour et al. demonstrated that IL6 and STAT3 overexpression increased nervous regeneration in the peripheral nerve through an increase of the nervous function and nervous velocity, histologically certifying stimulation of myelination. In addition, an increase in the number of nervous filaments was reported.

Our results are in concordance with these data that consider the indirect effects of fat tissue addition on muscle volume, confirmed by means of muscle ultrasonography. The particularity of our study, that may be regarded as an original finding was that of reporting that the dynamic variability of GFAP at 4 and at 10 weeks post-injury correlated with the addition of adipose tissue. This aspect has not yet been reported in literature to our knowledge, nor has the variability in GFAP expression in tandem with NFAP.

NFAP expression in the regenerated nerve has been somewhat more intensely studied compared to that of GFAP, especially in models that associate the addition of tubular structures as a guiding method for nervous regeneration (7). Variability in NFAP expression in peripheral nerve regeneration has been little explored, as a paradox of the evaluation of nervous regeneration. Similarly to our study, Carriel et al. observed that NFAP expression intensity in the regenerated nerve was much lower in comparison to the normal nerve (14). Most studies make use of growth-associated protein 43 (GAP43), which, in their opinion, is more specific for newly formed axons that do not yet express neurofilaments. Based on these data and on our observations, we may state that NFAP-positive neurofilaments were observed late. This denotes the fact that at 10 weeks after surgical injury, neurofilaments are mature and may partially sustain motor activity. The divergent GFAP and NFAP expression at 4 and 10 weeks reported in our study may also be considered an element of originality. Regarding this divergent expression, Triolo et al. noted that the disappearance or the absence of GFAP is balanced by the appearence of intermediate vimentin filaments and neurofilaments in the regenerated axon (15). The mechanisms through which this variability occurs are not yet fully understood and even less reported in the case of adipose tissue addition.

The GFAP reaction was at a maximum level at 4 weeks after injury. A decrease in the number and intensity of GFAPpositive cells at 10 weeks was noted. The number of nervous threads increased at 10 weeks. The regeneration of the nervous threads appears to be chaotic at 10 weeks. Is this a remodeling process? The regeneration phenomenon is incomplete.

Differential assessment in early (4 weeks) and late (10 weeks) stages of nerve regeneration may improve the understanding of microscopic changes responsible for incomplete nerve regeneration as an answer for several controversies from the literature. 
Based on our findings, we conclude that nerve regeneration is a multistep process, with time-dependent differential ability of reactive Schwann cells and neurons to recover. Reactive Schwann cells have the highest involvement in the early stages of nerve regeneration, while neuronal component recovery appears later and is predominant in the late stages of nerve regeneration. This may influence the future cellular and functional steps of nerve regeneration, explaining, in part, the heterogeneity of nerve regeneration after injury.

\section{References}

1 Memoli VA, Brown EF and Gould VE: Glial fibrillary acidic protein (GFAP) immunoreactivity in peripheral nerve sheath tumors. Ultrastruct Pathol 7: 269-275, 1984.

2 Salazar MF, Tena Suck ML, Rembao Bojórquez D and Salinas Lara C: Intraventricular Neurilemmoma (Schwannoma): Shall GFAP immunostaining be regarded as a histogenetical tag or as a mere histomimetical trait? Case Rep Pathol 2016: 2494175, 2016

3 Fu X, Tong Z, Li Q, Niu Q, Zhang Z, Tong X, Tong L and Zhang $X$ : Induction of adipose-derived stem cells into Schwannlike cells and observation of Schwann-like cell proliferation. Mol Med Rep 14: 1187-1193, 2016.

4 Bombeiro AL, Santini JC, Thomé R, Ferreira ER, Nunes SL, Moreira BM, Bonet IJ, Satori CR, Verinaud L and Oliverira Al: Enhanced immune response in immunodeficient mice improves peripheral nerve regeneration following axotomy. Front Cell Neurosci 10: 151, 2016

5 Lasso JM, Pérez Cano R, Castro Y, Arenas L, García J and Fernández-Santos ME: Xenotransplantation of human adiposederived stem cells in the regeneration of a rabbit peripheral nerve. J Plast Reconstr Aesthet Surg 68: e189-197, 2015.

6 Nurkovic J, Dolicanin Z, Mustafic F, Mujanovic R, Memic M, Grbovic V, Skevin AJ and Nurkovic S: Mesenchymal stem cells in regenerative rehabilitation. J Phys Ther Sci 28: 1943-1948, 2016.

7 Ghoreishian M, Rezaei M, Beni BH, Javanmard SH, Attar BM and Zalzali H: Facial nerve repair with Gore-Tex tube and adipose-derived stem cells: an animal study in dogs. J Oral Maxillofac Surg 71:577-587, 2013.
8 Berg A, Zelano J, Pekna M, Wilhelmsson U, Pekny M and Cullheim S: Axonal regeneration after sciatic nerve lesion is delayed but complete in GFAP- and vimentin-deficient mice. Plos One 8: e79395, 2013.

9 Nona SN, Duncan A, Stafford CA, Maggs A, Jeserich G and Cronly-Dillon JR: Myelination of regenerated axons in goldfish optic nerve by Schwann cells. J Neurocytol 21: 391-401, 1992.

10 Knoops B, Ponsar C, Hubert I and van den Bosch de Aguilar P: Axonal regeneration after peripheral nerve grafting and fibrinfibronectin-containing matrix implantation on the injured septohippocampal pathway of the adult rat: a light and electron microscopic study. Restor Neurol Neurosci 5: 103-117, 1993.

11 Lee HK, Seo IA, Suh DJ, Hong JI, Yoo YH and Park HT: Interleukin- 6 is required for the early induction of glial fibrillary acidic protein in Schwann cells during Wallerian degeneration. J Neurochem 108: 776-786, 2009.

$12 \mathrm{Li}$ J, Chen Y, Chen Z, Huang Y, Yang D, Su Z, Weng Y, Li X and Zhang X: Therapeutic effects of human adipose tissue-derived stem cell (hADSC) transplantation on experimental autoimmune encephalomyelitis (EAE) mice. Sci Rep 7: 42695, 2017.

13 H.Ashour, L.A.Rashed and M.M. Sabry: IL-6/STAT3 growth signalling induced by exercise promotes regeneration of injured rat sciatic nerve: return to an old cytokine conditioning. Comparative Exercise Physiology 13: 45-52, 2017.

14 Carriel V, Garzón I, Alaminos $\mathrm{M}$ and Cornelissen M: Histological assessment in peripheral nerve tissue engineering. Neural Regen Res 9: 1657-1660, 2014.

15 Triolo D, Dina G, Lorenzetti I, Malaguti M, Morana P, Del Carro U, Comi G, Messing A, Quattrini A and Previtali SC: Loss of glial fibrillary acidic protein (GFAP) impairs Schwann cell proliferation and delays nerve regeneration after damage. J Cell Sci 119: 3981-3993, 2006.
Received March 25, 2017

Revised April 13, 2017

Accepted April 19, 2017 